\title{
Arthur Conan Doyle y su detective científico*
}

\section{Clemens Franken K.**}

\section{Resumen}

Este artículo analiza la figura del famoso detective Sherlock Holmes, creado por Arthur Conan Doyle, bajo la perspectiva de su método de investigación, asociado a las ciencias positivistas predominantes en las últimas décadas del siglo XIX. A través* de una precisa observación, el detective se centra en criterios lógicos y abductivos para descubrir la verdad. Por lo tanto, los datos observados preceden a la teoría y el misterio cobra protagonismo en relación al crimen.

Palabras clave: Arthur Conan Doyle, abducción, conocimiento, observación.

\section{Arthur Conan Doyle And his Detective SCIENTIST}

\begin{abstract}
This article analyzes the world-famous detective Sherlock Holmes, created by Arthur Conan Boyle, under the perspective of his method of investigation associated with positivist science that prevailed in the last decades of the 19th century. Through precise observation, the detective focuses on logic and abductive reasoning to uncover the truth. Therefore, the observed data precedes the theory and the mystery takes a leading role in relation to the crime.
\end{abstract}

Key Words: Arthur Conan Doyle, abductive reasoning, knowledge, observation.

Recibido: 03-03- 2014Aceptado: 07-01-2015

* $\quad$ Este trabajo se enmarca en el Proyecto Fondecyt Regular No.1130218: Narrativa policial universal clásica, negra y posmoderna.

** Chileno. Doctor en Estudios Americanos Universidad de Santiago de Chile, Santiago, Chile, Profesor titular de la Pontificia Universidad Católica de Chile, Santiago, Chile. cfranken@uc.cl 


\section{Introducción}

Arthur Conan Doyle crea, casi medio siglo después de la aparición de Dupin, el detective de E. A. Poe, la famosa figura del detective Sherlock Holmes. El autor, originario de una familia irlandesa católica de presunta nobleza ${ }^{1}$ y educado por jesuitas, perdió su fe mientras estudiaba medicina, pues aprendió a buscar la verdad a través de la observación exacta, el análisis lógico y los experimentos. Ya durante los años en Southsea, donde hizo su práctica como joven médico, entró en conocimiento de ideas espiritistas que reemplazaban su religión y que estudió profundamente durante varias décadas (cfr. Buchloh y Becker 58).

Dado que su consulta médica nunca se volvió absorbente, Doyle contaba con tiempo disponible para retomar sus intentos literarios infantiles y juveniles, esta vez escribiendo ya no poemas ni relatos (Cfr. Skreb 38) sino novelas. En 1887, con Estudio en escarlata, y, tres años más tarde, con El signo de los cuatro, Doyle logró sólo cierto reconocimiento literario, pero un casi inmediato reconocimiento económico (Rzepka, 118). La figura del detective por sí misma se hizo popular² a través de los primeros cuentos detectivescos recopilados en los tomos Las aventuras de Sherlock Holmes (1891) y Las memorias de Sherlock Holmes (1893). La celebridad del personaje provocó que los lectores de la época nunca aceptaran la muerte del protagonista-detective, descrita por el autor en el último cuento del segundo tomo titulado El problema final, motivo por el cual Conan Doyle se vio obligado prácticamente a restringir la escritura de novelas históricas, a las cuales se sentía realmente llamado, y seguir publicando en el Strand Magazine, cada cierto tiempo, otros cuentos con Sherlock Holmes como protagonista. Fruto de este retorno medio forzado pero duradero, son otros tres tomos de cuentos: La reaparición de Sherlock Holmes (1905), Su último saludo en el escenario (1917) y, tres años antes de morir Doyle, Sherlock Holmes sigue en pie (1927). ${ }^{3}$

1 Según Paul G. Buchloh y Jens P. Becker, los Conan Doyle pueden remontar "ihren Stammbaum auf die Platagenets" ("su arbol genealógico [...] hasta los Plantagenets") (58).

2 Según John Scaggs, el "enormous success" ("éxito enorme") de los cuentos con Sherlock Holmes como protagonista se debe, en gran medida, a las "conservative middle-class attitudes towards crime and the maintenance of social order [...] The ideology is clear. Crime will always be punished, either by the law or by divine providence. The only exceptions to this rule are the sins of the aristocracy, whom Holmes either cannot, or will not, bring to justice". ("posturas conservadoras de la clase media frente al crimen y la mantención del orden social [...]. La ideología es clara. El crimen siempre será castigado, ya sea por la ley o por la providencia divina. La única excepción a esta regla son los pecados de la aristocracia, a la cual Holmes no puede o no quiere llevar ante la justicia") (25).

3 Respecto a los datos bio-bibliográficos de Arthur Conan Doyle confróntense, por ejemplo, las obras de Nusser (96-97), Del Monte (106-107) y Skreb (38-40). 
Según Alberto del Monte,

Sherlock Holmes había sido ideado feo: alto, delgado, desmadejado, con una nariz grande y ganchuda y unos ojos penetrantes y casi juntos. Pero después, el escritor se dejó influir por el ilustrador, quien, por su parte, había interpretado el gusto del público, y el detective adquirió su fisonomía definitiva. Se convirtió en un tipo guapo y atlético, caracterizado por su rostro enjuto, por su cráneo dolicocéfalo y sus manos finas; por las orejeras de su gorro de viaje y por su bata; por la pipa y la jeringa para inyecciones de morfina; [...]. (107-108)

En su primera novela, Estudio en escarlata, Doyle revela otros antecedentes de Sherlock Holmes, quien reconoce que él es un "aficionado [tanto] a la observación como a la deducción" lo que, junto con su "conocimiento de la historia criminal" (27), lo capacita para dar solución a cualquier enigma policial. Por otra parte, pese a que de "[d]e literatura contemporánea, filosofía y política, estaba casi completamente en ayunas"4 e, incluso, que "ignoraba la teoría copernicana y la composición del sistema solar" (21), sí tenía profundos estudios en disciplinas de las Ciencias Naturales, "inmensos" conocimientos de "literatura sensacionalista", tocaba bien "el violín" y era experto "boxeador y esgrimista de palo y espada" (23). Pero, ante todo, "Holmes posee un carácter demasiado científico" para el gusto de Watson, y "habita en su persona la pasión por el conocimiento detallado y preciso" (13).

Al igual que Poe, Doyle inicia su actividad como escritor de novelas policiales con una exposición teórica que titula "La ciencia de la deducción". En ella opina lo siguiente de Poe y su detective:

Dupin era un tipo de poca monta. Ese expediente de irrumpir en los pensamientos de un amigo con una frase oportuna, tras un cuarto de hora de silencio, tiene mucho de histriónico y superficial. No le niego, desde luego, talento analítico, pero dista infinitamente de ser el fenómeno que Poe parece haber supuesto. (28-29)

4 No obstante, como afirma Manuel Valle, "en numerosas ocasiones, Holmes se presenta como una persona de excelente formación, que conoce y cita a Petrarca, Eckermann, Goethe, Flaubert, George Sand, Shakespeare, Boileau, La Rouchefoucauld, etc., [y] que acude a conciertos de Sarasate o Norman-Neruda para escuchar música de Wagner" (43). 
En sus memorias, sin embargo, Doyle reconoce la influencia de Poe en su narrativa policial y expresa su admiración por él, como también por W. Collins, C. Dickens y E. Gaboriau 5 . De hecho, Doyle funde en su obra "las dos corrientes de la tradición policíaca, la de la aventura y la de investigación racional" (Del Monte 110-11). De esta forma, logra satisfacer las exigencias de un amplísimo público y cimenta la base de su popularidad, la que encontró su máxima expresión en el hecho de que Sherlock Holmes "dejó de ser un personaje literario, se convirtió en una criatura viviente ${ }^{6}$ y borró la memoria de su creador"7 (Del Monte 111).

Manfred Smuda, desde una perspectiva estético-recepcionista, ve justamente en el hecho de la acomodación al gusto del público el pecado de Conan Doyle que convierte sus cuentos en textos triviales:

Das Modell der Geschichte 'The Murders in the Rue Morgue'
mit dem Glauben an die Leistungsfähigkeit der raticination
bleibt für Conan Doyle der Idealtypus der Detektivgeschichte.
Was bei ihm variiert, ist lediglich attributiver Natur in bezug
auf dieses als einzigartig anerkannte Modell. In dieses werden
immer nur neue Inhalte gegossen, ohne dass es selbst variiert
wird. Wenn aber in ein stereotypes Schema bloss neue
inhaltliche Sachverhalte eingeführt werden, passt sich die
Detektivgeschichte dem Erwartungshorizont des Lesers an;

5 John Scaggs habla de "a clear debt to the novels of Gaboriau" ("una deuda clara a las novelas de Gaboriau") y de "Doyle's debt to The Moonstone" ("la deuda de Doyle a The Moonstone") de W. Collins (24). Por su parte, Symons señala que "Doyle no doubt took the ideas for some of these disguises from Vidocq, as he took Holme's style of interpreting thoughts from Poe and the form of some deductions from Gaboriau" (sin lugar a dudas, Doyle tomó las ideas de algunos de los disfraces de Vidoq, como también el estilo de Holmes de interpreter los pensamientos de Poe y la forma en que realizaba algunas deducciones de Gaboriau) pese a lo cual concede el mérito creador del autor al reconocer que "Holmes is conceived in outline, with attributes that are really substitutes for characterization -the eagle eye, the misanthropy, the remoteness. It is a mark again of Doyle's skill that Sherlock Holmes comes through to us as a man who genuinely had a genius for his occupation" ("Holmes es concebido en líneas generales, con atributos que son realmente sustitutos de una caracterización -los ojos de lince, la misantropía, la lejanía. Una vez más es una marca de la habilidad de Doyle que Sherlock Holmes nos aparezca como un hombre que genuinamente era un genio en su profesión") (Symons 71).

6 Se discutió incluso entre los lectores la fecha de su nacimiento y la Sherlock Holmes Society de Londres la fijó "en el 15 de enero de 1854", mientras que los Baker Street Irregulars la adelantan al "8 del mismo mes". "En 1954 se ha celebrado su centenario. Su piso del núm. 221b de Baker Street, Marylebone, reconstruido hasta en sus más mínimos detalles, es meta ininterrumpida de visitantes" (Del Monte 111).

7 Aunque "a partir de sus diferencias con Dupin, Sherlock Holmes se configura como un personaje 'distinto', dotado de unas características propias", para Manuel Valle "el truco de romper el pensamiento que aparece en Los crímenes de la calle Morgue es considerado como petulante y superficial, pero en otros relatos Sherlock Holmes hará exactamente igual con Watson, lo que demuestra que una cosa son las opiniones de Holmes y otra lo que realmente hace Conan Doyle" (55). Además, al igual que el detective de Poe, es capaz de ponerse en el lugar del otro y, a través de la imaginación, predecir los comportamientos de los demás (McInerny). 


\section{denn der stereotype Rahmen der Geschichten garantiert ein immer gleiches Lösungsschema des Problems. [...] Damit aber nimmt die Detektivgeschichte eine Richtung auf 'perfekte Banalität'. Weil sie sich den Erwartungen des Lesers nahezu völlig anpasst, wird sie trivial. $(47)^{8}$}

Aunque, por un lado, se justifica esta crítica y se echa de menos en sus relatos policiales una corriente subterránea de sentido a la manera de Poe, hay que reconocer, por otro lado, que Doyle logra presentar las operaciones intelectuales de Sherlock Holmes en forma tan entretenida que pierden la rigidez de tratado científico que suelen tener en Poe, ante todo, en su primer cuento policial. Además, la permanente repetición de determinadas situaciones como, por ejemplo, las escenas iniciales ${ }^{9}$ de

8 "El modelo del cuento 'The Murders in the Rue Morgue' con su fe en la eficacia del raciocinio se mantiene en Doyle como el tipo ideal del relato policial. Lo que varía en él es sólo de segundo orden en relación a un modelo reconocido como único. Este modelo se llena siempre sólo con nuevos contenidos sin variarlo. Pero cuando se introducen únicamente nuevas cosas en un esquema estereotipado, el cuento policial se adapta al horizonte de expectativa del lector, pues el marco estereotipado de los cuentos garantiza siempre el mismo esquema de solución del problema.[...] De esta forma, el relato policial se encamina en dirección a la 'banalidad perfecta'. Porque se adapta casi completamente a las espectativas del lector, se convierte en un texto trivial" (47).

9 Según Viktor Schklovskij, el esquema detallado de los cuentos policiales de Conan Doyle es el siguiente:

I. Erwartung, Gespräch über frühere Fälle, Analyse.

II. Auftritt eines Klienten.

Hauptteil der Erzählung:

III. Indizien, die in der Erz"hlung angeführt werden. Am wichtigsten sind zweitrangige Angaben, die so eingebaut sind, dass der Leser sie nicht zur Kenntnis nimmt. Hier eben wird das Material für eine falsche Lösung geboten.

IV. Watson gibt den Indizien eine falsche Deutung.

IV. Watson gibt den Indizien eine falsche Deutung.

V. Fahrt zum Tatort [...].

VI. Der beamtete Detektiv gibt eine falsche Lösung; wenn kein Detektiv vorhanden ist, dann wird die falsche Lösung von einer Zeitung geboten, von den Leidtragenden oder von Sherlock Holmes selbst. VII. Die Zwischenzeit wird ausgefüllt mit Gedanken Watsons, der nicht versteht, worum es geht. Sherlock Holmes raucht oder macht Musik. Manchmal fasst er die Fakten in Gruppen zusammen, ohne eine endgültige Schlussfolgerung zu ziehen.

VIII. Die Lösung ist fast immer unerwartet. Um sie herbeizuführen wird sehr oft ein Mordanschlag, den jemand vorhat, ausgenützt.

IX. Analyse der Fakten durch Sherlock Holmes.

I. Espera, conversación sobre casos anteriores, análisis.

II. Aparición de un cliente.

Parte principal del relato:

III. Indicios que son mencionados en el relato. Los más importantes son los datos secundarios que son incorporados para que el lector no los note. Aquí se ofrece el material para una solución errónea.

IV. Watson les da una interpretación falsa a los indicios.

V. Viaje al lugar de los hechos [...].

VI. El detective-policía da una solución falsa; si no hay un detective-policía la solución falsa es presentada por un diario, un afectado o por Sherlock Holmes mismo.

VII. El tiempo entremedio se llena con ideas de Watson que no entiende de que se trata. Sherlock Holmes fuma o hace música. A veces resume los hechos parcialmente sin sacar una conclusión definitiva.

VIII. La solución es casi siempre inesperada. Para provocarla se aprovecha a menudo un intento de asesinato planeado.

IX. Análisis de los hechos por parte de Sherlock Holmes (93-94). 
los cuentos de Doyle o la forma como se analizan los hechos, producen placer estético en el lector regular de los cuentos de Sherlock Holmes y aseguran así su popularidad. A pesar de que el resumido esquema de todos los relatos de Conan Doyle es el mismo: "misterio, réplica de Sherlock Holmes, contraofensiva, a veces, del criminal, victoria de Sherlock Holmes, explicaciones finales" (Boileau y Narcejac 56), no es menos cierto que a menudo se menosprecia la calidad de la escritura de este autor inglés, "su claridad, su sobriedad, la seguridad con que narra, la amenidad de su estilo" (Boileau y Narcejac 56). Kayman confirma lo anteriormente dicho destacando por su parte que el éxito de la fórmula creada por Doyle se debe a "the verve of Doyle's writing, the ingenuity of the stories and the skill and economy of their construction" que producen, según él, "fundamental narrative satisfactions" (48). ${ }^{10}$

\section{Doyle y una tercera vía para encontrar la verdad: la abducción}

Lo que nadie puede quitarle a Conan Doyle es la creación de una nueva imagen literaria y, como afirma Rainov, "la invención de un personaje es, quizás, el acto creador más difícil" (56). El hecho de que Doyle haya creado y modelado la legendaria figura de su detective Sherlock Holmes inspirándose no sólo en Dupin, sino, además, "en su profesor, el doctor Joseph Bell, miembro del Royal Infirmary de Edimburgo", indica su "intento plenamente consciente de introducir en la indagación criminal un método de mayor rigor científico que el que se había usado hasta entonces" (Sebeoc y Umiker-Sebeoc 54).

Queda así claramente establecido que nos encontramos en la época positivista de las últimas décadas del siglo XIX, cuando las ciencias exactas forman el modelo de la ciencia por imitar. Pensemos solamente en Emile Zolá que, en 1880, siete años antes que Conan Doyle, en su ensayo sobre $L a$ novela experimental, pretende aplicar el método científico-experimental del médico Claude Bernard a la literatura. Mientras que Poe incorporaba la imaginación poética en su raciocinio, rescatándola de esta forma, el teórico naturalista Zolá y el positivista Doyle rinden culto al modelo de las ciencias exactas, imitándolas. Doyle, para Rzepka, fue el primer escritor

10 "[L]a vivacidad de la escritura de Doyle, la ingenuidad de los cuentos y la habilidad y economía de sus construcciones" "fundamentales satisfacciones narrativas". 
de la ficción de detectives "to conceive the plot of detection specifically in this way, as a device for uncovering a hidden narrative 'idea' through the imaginative projection of analeptic arrays invoked by 'lay[ing] emphasis on everything' suggesting 'alternative' explanations. Only one of these, the one arrived at by the detective, would turn out to be correct"11 (Rzpeka 119). Por eso, las hipótesis de Holmes se "apoyan en los sólidos códigos referenciales propios de determinadas ciencias experimentales o de ciertos sectores bien consolidados e institucionalizados de las ciencias experimentales" (Bonfantini y Proni 174), que se cuentan, como vimos más arriba, entre el tesoro de conocimientos de Holmes.

Lo peculiar y, al mismo tiempo, popular del modus procedendi de Holmes confluye desde dos vertientes. En primer lugar, se fundamenta en el hecho de que el estilo de sus deducciones obedece tanto "a un imperativo de simplicidad y de plausibilidad según criterios lógicos y empíricos firmemente establecidos en la sociedad" (176). En segundo lugar, se basa en "una prohibición complementaria: no pretender adivinar jamás" (176).

Para Holmes, el punto de partida en cualquier investigación policial, siguiendo a su maestro Joseph Bell y basándose en la idea popularizada del procedimiento de las Ciencias Naturales, es siempre una aguda y precisa observación. En la ya citada parte teórica-introductoria de su primera novela policial, dice Sherlock Holmes al respecto, textualmente: "La capacidad de observación constituye en mi caso una segunda naturaleza" (28). Mientras que Dupin resolvía los problemas meditando en su gabinete de ensueño o luego de una breve visita al lugar de los hechos, Holmes investiga detalladamente todos los indicios, usando lupa y cinta métrica. Por eso, Holmes da énfasis en su "[i]mparcialidad y receptibilidad para los datos" y "en el hecho de que él 'observa' lo que otros meramente 'veían'" (Truzzi 93). Holmes tiene conciencia de que el diablo se esconde tras el detalle, que no hay nada más engañoso que un hecho obvio y que las cosas pequeñas son las más importantes. Los

11 “[E]n concebir la trama de la investigación como tal, como un artefacto para descubrir una 'idea' narrativa oculta a través de la proyección imaginativa de la diversidad analéptica invocada al 'poner énfasis en todo' sugiriendo explicaciones 'alternativas'. Solo una de estas, aquella a la que llega el detective, se transformaría en la correcta". 
datos observados preceden, por lo tanto, a la teoría. La observación12 de Holmes no incluye, sin embargo, solamente los hechos y acontecimientos, sino también la ausencia de ellos ${ }^{13}$.

Abase de la observación de los hechos y datos, minucias y trivialidades, Holmes construye ahora sus deducciones teóricas que poseen, en general, "una aparente lógica rigurosa" (Boileau y Narcejac 54). Es importante comprender bien la relación entre hechos y deducción, considerando a esta última como una inferencia de hechos observados a hechos no observables, ocultos, pero acaecidos o que acaecerán sugeridos por las observaciones. Por ejemplo, en el cuento "El enemigo de Napoleón", Holmes deduce que el criminal buscaba algo a partir de la observación de que éste se tomó la molestia de llevar el busto adonde pudiese examinar sus pedazos.

Hay que señalar aquí que el método de Holmes no es propiamente deductivo (de lo general a lo particular) ni inductivo (de lo particular a lo general), sino abductivo. Según Bonfantini y Proni, las hipótesis individuales de Holmes, los pasos individuales de su compleja 'trama' retroductiva, pueden clasificarse en una de las cuatro clases siguientes:

1. Se apoyan en los sólidos códigos referenciales propios de determinadas ciencias experimentales [...] que [...] se cuentan entre el sólido bagaje de conocimiento de Holmes

12 Sonja Osterwalder agrega respecto a la observación un detalle muy interesante: “Geradezu frenetisch gefeiert wird in Conan Doyles Geschichten die Physiognomik, wenngleich die expliziten Erwähnungen des Wissenschaftszweiges unterbleiben; doch dass ein enger Zusammenhang zwischen Aussehen und charakterlicher Anlage besteht, scheint auf fast jeder Seite auf. Auch die Phrenologie feiert immer wieder Auftritte [...]".

("Casi frenéticamente se celebra en los cuentos de Conan Doyle la fisiognomía, a pesar de que no se menciona dicha rama científica; sin embargo, que exista una relación entre apariencia física y carácter se trasluce casi en cada página. También aparece la frenología [...]"). (2011:66)

Esta lúcida afirmación de Osterwalder confirma nuevamente la cercanía de Conan Doyle con E. A. Poe.

13 Para McInerny las hipótesis de Holmes se crean en un proceso de cuatro etapas, que se inician con la observación y la reunión de datos:

[t]here is first the fact-gathering stage which consists in two steps: the interview and the on-thescene investigations. The second stage is the one in which Holmes gives himself over to intense thought, analyzing the data that has been gathered and forming whatever conclusions the data will warrant. The third stage [...] is where he solves the mystery and closes he case. The fourth and final stage is the after-the-fact briefing, in which Holmes ties up loose ends and answers any lingering questions concerning the case.

("primero está la etapa de reunión de hechos, que consiste en dos pasos: la entrevista y la investigación de la escena. La segunda etapa es en la que Holmes se vuelca al pensamiento intenso, analizando los datos reunidos y formando la conclusión sugerida por esos datos. La tercera etapa es en la que Holmes resuelve el misterio y cierra el caso. La cuarta y final etapa es el informe post factum, en la que Holmes ata cabos sueltos y contesta preguntas concernientes al caso"). 
(botánica, geología, química, anatomía; y podemos añadir fisiología y semiótica médica).

2. Se apoyan en sólidos códigos referenciales pertenecientes a áreas especiales de conocimiento y de técnicas de clasificación e identificación que el propio Holmes ha elaborado y reorganizado [...] en monografías sobre temas tales como la manera de distinguir tipos de cenizas según los tabacos, de sacar moldes de huellas y [...]. ${ }^{14}$

3. Se apoyan en vastos y bien organizados repertorios de conocimiento descriptivo concerniente a los usos de la vida cotidiana.

4. Se apoyan en el simple sentido común y corriente o en el saber común relativo a la lógica del comportamiento.

Basta citar ciertos ejemplos de STUD [Estudio en escarlata; C.F.]. La hipótesis de que la víctima había sido envenenada pertenece evidentemente a la clase 1; la hipótesis relativa al calzado del asesino, al igual que la proverbial afirmación de que 'el asesino fumaba un cigarro Trichinopoly', pertenecen obvia-mente a la clase 2; la identificación de que la distancia entre ruedas correspondía a un coche de punto, pertenece a la clase 3; la sospecha respecto a Jefferson Hope, sabiendo que la víctima había pedido protección contra él y que J.H. había tenido rivalidades amorosas con la víctima, pertenece por supuesto a la clase 4. (174)

Para comprobar estas afirmaciones tomamos otros ejemplos, esta vez de la novela El sabueso de los Baskerville. El escenario del acontecer se traslada desde Londres hacia una localidad rural cerca de Devonshire, matizando la acción con el lóbrego paisaje campestre de la zona. La investigación pretende, más que evitar un nuevo asesinato, desentrañar mediante el uso de la razón un móvil criminal común oculto en una investidura paranormal. Frente a la incertidumbre del caso, el detective debe crear una serie de conjeturas que comprobará o refutará a lo largo de la investigación. Cuando el Dr. Mortimer le señala a Holmes que se están adentrando en el terreno de las suposiciones, el investigador

14 Con razón, Kaymann llama a Holmes “a self-styled expert” (“un experto a su propio estilo”) (50). 
replica: "Diga más bien en la región donde sopesamos las probabilidades y elegimos la más factible. Es el uso científico de la imaginación, pero siempre disponemos de algunas bases materiales para iniciar nuestras especulaciones" (43). Dicho con otras palabras, la conjetura, la invención de hipótesis acerca de las causas desconocidas de los hechos observables, es decir, lo que Umberto Eco ${ }^{15}$ y otros lingüistas y/o semiólogos llaman abducción, constituye un momento decisivo de la investigación de Holmes.

En esta novela, encontramos justamente los ya mencionados cuatro tipos de abducciones. Un elemento que le revela a Holmes el móvil real del crimen es obtenido mediante una abducción de la primera clase: al contemplar un retrato familiar de los Baskerville, el detective reconoce a Stapleton como pariente de Sir Henry mediante un análisis de sus rasgos físicos, dado el gran parecido que éste posee con quien dio inicio a la maldición familiar. Holmes realizas una abducción de la segunda clase cuando establece que las letras recortadas con la que se confeccionó la carta anónima recibida por Sir Henry correspondían al diario Times del día anterior, ya que reconoció el tipo de imprenta bourgeois característica de dicho periódico. Según Holmes, "[e]l conocimiento de los tipos de imprenta es una de las más elementales ramas del conocimiento del especialista en delitos" (42). Del mismo modo, Holmes comprobó la participación de una mujer en la intriga, debido al perfume de jazmín blanco que percibió en la carta, lo cual lo condujo a sospechar de los Stapleton y hacer el siguiente comentario a Watson: "[h]ay setenta y cinco perfumes que el criminalista debe ser capaz de distinguir, y, en mi propia experiencia, en más de una ocasión ha habido casos cuya solución ha dependido de un reconocimiento rápido de dichos perfumes" (192). Con relación a la misma carta, Holmes realiza una abducción de la tercera clase cuando señala que la carta fue elaborada en un hotel, dado que la única palabra escrita poseía tales imperfecciones en su caligrafía que debía haberse realizado con una pluma y un tintero en mal estado, algo propio de esos instrumentos que se encuentren en las habitaciones de un hotel. Y, finalmente, Holmes realiza una abducción de la cuarta clase

15 Para Umberto Eco, que distingue tres tipos de abducciones en su artículo "Cuernos, cascos, zapatos: Algunas hipótesis sobre tres tipos de abducción" (El signo de los tres. Dupin, Holmes, Peirce.265-294), "[m]uchas de las llamadas 'deducciones' de Sherlock Holmes son casos de abducción creativa" (287). 
cuando afirma que las pisadas dejadas por Sir Charles no eran las de una persona que caminaba en puntillas, sino las de una que estaba corriendo. ${ }^{16}$

Podemos considerar el hecho de que Holmes valida en sus diferentes tipos de abducciones la imaginación como medio para descubrir la verdad, aunque, a diferencia de Dupin, esta requiera de un soporte empírico en una primera instancia y "Holmes exi[ja] después una ratificación empírica de las hipótesis resultantes en términos que se aproximan mucho a lo que hoy se llama método hipotético-deductivo" (Truzzi 97).

Sin embargo, es necesario mencionar que la utilización de esta determinada metodología sugiere varios aspectos de importancia. Por un lado, como Bagget afirma, en la práctica detectivesca es condición sine qua non la valentía a la hora de probar y experimentar. De tal modo, las equivocaciones de Holmes y su vanidad y soberbia no son otra cosa que muestra de su "coraje epistemológico" a la hora de aventurar hipótesis y ponerlas a prueba:

[h]is willingness to give it a shot [...] is both interesting and important. It's instructive because it illustrates, at its best, an epistemic virtue. To get at the idea, consider someone racked with so many doubts about his intellectual abilities, that he becomes debilitated and diffident, never or hardly ever willing to risk an inference [...] [Holmes], [1]ike a good scientist [...] put his ideas to the test, weighed them on the scales of balancing probabilities ${ }^{17}[1]$.

Existen, no obstante, otras visiones con respecto al tema. Aunque reconoce que el método de Holmes tiene "un enorme valor para las

Cawelti destaca, también, esta novela de Doyle, porque ella presenta un ejemplo definitivo del anuncio de la solución del misterio por parte del detective, transformándolo en un aspecto incluso más importante que la propia aprehensión del criminal. Para Cawelti, el drama y suspenso contenido en la escena en que Watson cree estar a punto de capturar al criminal y, en cambio, se encuentra cara a cara con Holmes, crea un paradigma en este tipo de literatura porque contrasta finalmente al "frantic narrator who is hopelesly lost in the maze of clues and testimony and the supremely calm detective who now takes the action into his hands"(el agitado narrador que está perdido sin esperanzas en el laberinto de pistos y testimonios y el detective supremamente calmado que ahora toma la acción en sus manos). De esta forma, "what seemed chaotic and confused is revealed as clear and logical" ("lo que parecía caótico y confuso se revela como claro y lógico") (Cawelti 87).

17 "[S]u disposición para intentarlo [...] es, a la vez, interesante e importante. Es instructiva porque, en su mejor variante, ilustra una virtud epistémica. Para comprender la idea, considere a alguien aquejado con tantas dudas sobre sus habilidades intelectuales, que se vuelve débil, incapaz o casi de arriesgar una inferencia [...] Holmes, como un buen científico, prueba sus ideas y contrapesa las probabilidades". 
disciplinas de las que procede y en las que se encuadra", para Manuel Valle, "el interés del relato no puede residir nunca en el método científico / deductivo empleado" (255). Sin necesariamente compartir la opinión del autor, es evidente que en la construcción narrativa de los cuentos y novelas de Sherlock Holmes, tiene gran importancia "[1]a definición del relato policial como 'relato de misterio' [puesto que] separa la 'narración' de la 'investigación' del caso. El núcleo literario del relato no reside en la manera [...] en que se lleva a cabo la investigación [...], sino en la forma [...] en que se construye el misterio". Así, "tal como lo practica Holmes continuamente (la famosa 'teatralidad' del detective) y, sobre todo, tal como lo produce Watson en sus relatos, el misterio [...] es un 'efecto sorprendente', producido 'en -y por- el propio texto'”' (256).

Aparte de la capacidad principal y decisiva de observar, deducir o más bien abducir, Holmes se caracteriza también por sus amplios conocimientos, dado que cuenta con una vasta gama de elementos de información especializados y útiles en su área de trabajo. Además, él era un sistemático coleccionista de casos históricos, así como de episodios famosos de insólita astucia o ingeniosidad. En el capítulo sobre su "Ciencia de la deducción", Holmes reconoce este hecho en las siguientes palabras dirigidas a Watson: "[s]abe usted que he atesorado una cantidad respetable de datos fuera de lo común; ese conocimiento facilita extraordinariamente mi tarea" (28). Sus conocimientos le ayudan, ante todo, para sus abducciones, es decir en el proceso de la elaboración de hipótesis y conjeturas, ya que en la presentación de cada caso nuevo surgen en él similares casos anteriores. Por ejemplo, en el ya mencionado cuento "El enemigo de Napoleón", Holmes pasó, según Watson, la tarde entera entre montones de periódicos viejos que atestaban su cuarto trasero y, cuando por fin bajó, tenían sus ojos un brillo de triunfo porque había encontrado una información acerca de antiguos casos criminales -específicamente el robo de la perla, ocurrido hacía bastantes meses atrás-, que puede relacionar con el caso actual a resolver.

\section{Ojo privado versus Ojo público: la postura crítica del detective}

En la elaboración de las hipótesis y de las inferencias a partir de las observaciones, tampoco están ausentes la imaginación y la intuición, tan fuertemente presentes en Dupin, pero quedan, al igual que el 
conocimiento, opacadas por el modelo de planteamiento racionalista y positivista18. El estilo sobrio y discreto que delata a un científico es la lógica consecuencia. En su "Ciencia de la deducción", Holmes sostiene estar en posesión de "una especie de intuición" (27) que lo capacita para resolver casos menos complejos a la manera de Dupin, es decir, "sin salir de esta habitación [...] [y sin] contacto directo con las cosas" (27). Es decir, el método de Holmes permite reconocer su parentesco con Dupin, su alma de "artist of the purest sort [...] Holmes is a typical nineteenthcentury aesthete in his devotion, his 'art for art's sake"'19. (Males cit. en Buchloh y Becker 63). El hecho de que Holmes "es de buena familia (desciende, por el lado materno, del pintor francés Vernet), trata de tú a tú a la alta sociedad" (Del Monte 110-11), fuma opio, toca el violín, es insensible al amor y, según Watson, un excéntrico, lo muestra como una mezcla de "Denker und Künstler, er besitzt die 'bi-part soul' des Dupin und repräsentiert"... den Typ des 'artist-philosopher'"20 (Buchloh y Becker 61).

Hasta qué punto Holmes es también un artista puede mostrarse muy bien en el cuento "La liga de los pelirrojos", donde es posible establecer un paralelo entre la metodología de investigación de Holmes y una composición musical. El texto señala, además, la afición y la destreza musical del detective Holmes, que incluso tararea una melodía escuchada en un concierto momentos previos a la captura del ladrón. La acción del detective es equiparada con la de un músico, en cuanto la investigación se construye sobre la base de un procedimiento armónico: las pistas deben configurarse como notas musicales sobre un pentagrama, las cuales, al ser ejecutadas, conducen a la resolución melódica del enigma. Esto recuerda el pensamiento pitagórico que hermana la música con la matemática, en cuanto ambas tienen como objetivo satisfacer una necesidad de orden en las acciones humanas.

18 No obstante, es innegable que la imaginación tiene un rol fundamental en la construcción y comprobación de las hipótesis. De tal forma, "[r]eality - the concrete circumstances of the casepresents him with the bare facts, but imagination must come into play in the interpretation of those facts" ("la realidad - las circunstancias concretas del caso- se le presenta con los hechos desnudos, por lo que la imaginación debe entrar al juego en la interpretación de esos hechos").

19 "[A]rtista de la especie más pura ... Holmes es un esteta típico del siglo diecinueve en su devoción, en su 'arte por el arte'".

20 "[P]ensador y artista, [que] posee la 'bi-part soul' de Dupin y representa el tipo del 'artistphilosopher"'. 
Por todo eso, sería un error considerar al personaje de Holmes sólo unidimensional, frío, científico ${ }^{21}$. Al final del cuento "El enemigo de Napoleón", hasta es capaz de conmoverse ante la alabanza del policía Lestrade:

-Muchos y muy difíciles asuntos os he visto resolver, Sr. Holmes -dijo Lestrade al cabo de un rato-; pero ninguno tan maravilloso ni tan admirable como éste. En Scotland Yard todos estamos orgullosos de que nos ayudéis en nuestras empresas; y si mañana os dignáis ir allá, desde el primer inspector hasta el último agente se disputarán el honor de estrecharos la mano.

Holmes volvió la cabeza para ocultar su emoción.

-Gracias, gracias - balbuceó.

Un segundo después había recobrado su sangre fría habitual [...]. $(235)^{22}$

En este mismo cuento, Holmes plantea también una crítica a los grupos sectarios de la sociedad londinense de la época. Puntualmente, existe una crítica subyacente a la francomasonería representada en el personaje Jobes Wilson, quien pertenece a esta secta y es víctima del actuar del delincuente, el cual se aprovecha de la confianza y de la credulidad del prestamista para planear un robo que se perpetraría a una entidad bancaria vecina al negocio del masón. La Liga de los pelirrojos simboliza una institución exclusiva que parece entregar un beneficio a un grupo limitado de personas, cuando en realidad lo que hace es aprovecharse de ellos para satisfacer sus propios intereses. Incluso se puede establecer

21 Cabe mencionar que la visión del mundo femenino como una dimensión casi puramente sentimental y anticientífico (con algunas excepciones), generó que la lectura de Holmes desde los estudios feministas pintara al personaje como un misógino empedernido.

22 Por otro lado, Valle enfatiza la falta de apego emocional que suele mostrar Holmes con respecto al otro. Tal vez sus puntos más sensibles sean su propio ego y su propia vanidad, pero en lo que implica relación con otros seres humanos, el detective mantiene su actitud fría y desafectada. Incluso evita las mujeres y los enredos amorosos para no intervenir en la actitud cientificista que lo hace observar todo su entorno -y también a otras personas-, como un objeto más, susceptible de ser analizado a través de un determinado método. En tal sentido, puede justificarse que Holmes "huya de los sentimientos, y especialmente del sentimiento amoroso, ya que éste significaría la imposición del sentimentalismo propio del objeto sobre el cientifismo necesario para la investigación criminal" (Valle 116). Sin embargo, desde el punto de vista de Bagget, la soberbia puede considerarse valentía y para McInerny no siempre vemos "how reason relates to passion in [Holmes] -as the ruler to the ruled. Here is a man of passion who manages, through the exercise of reason, to keep his passions continuously under nearly perfect control, and this offers the best explanation for the man's amazing efficiency" ("cómo la razón se relaciona con la pasión en Holmes- como el dominante se relaciona con el dominado. He aquí un hombre apasionado que se las arregla, a través del ejercicio de la razón, para mantener sus pasiones bajo un control continuo y casi perfecto, y esto ofrece la mejor explicación para su asombrosa eficiencia") (McInerny). 
una relación entre el oro que se pretende robar y la francomasonería, en cuanto ambos son de origen francés y el oro representaría el pensamiento iluminista que fundamenta a la ideología masona. De este modo, es posible constatar que Conan Doyle plantea una denuncia subterránea en contra de las instituciones sociales que, según él, poseen una dudosa base ideológica y pueden llegar a ser el punto de partida de organizaciones criminales. Un caso paralelo de crítica social encontramos en Estudio en escarlata, en la que Doyle-Holmes plantea también algo parecido de la secta religiosa de los mormones en el estado de Utah (EE.UU.).

Sin embargo, la crítica social de Sherlock Holmes se dirige, ante todo, en contra de la policía. A Holmes le molesta especialmente el hecho de que los inspectores de Scotland Yard desean la gloria y fama, adjudicándose la resolución de los enigmas que solamente lograron descifrar gracias a su ayuda. El arriba citado reconocimiento por parte del inspector Lestrade constituye, sin duda, una excepción. Tal vez por esta razón Holmes se conmovió.

Holmes le critica, a la policía en general, su falta de rigor metodológico y su carencia de capacidad de observación, ya que realiza conjeturas muy apresuradas. En su primera novela policial, Holmes se ríe, por ejemplo, de las conclusiones apresuradas de Lestrade, cuando este saca la conclusión de que debe de haber estado una mujer en la pieza y cuando, más tarde, para comprobarlo, atribuye a Rachel, nombre de mujer, la palabra Rache que encontró escrita en la pared (cfr. 41). Este método "del viejo perro de rastreo" le causa a Holmes un ataque de risa y él "desent[ierra] de su bolsillo una cinta métrica y una lupa, de grueso cristal y redonda armadura" (42). Luego de una investigación minuciosa les comunica a los atentos policías que el asesinato se ejecutó con "veneno" y de paso le dice a Lestrade antes de salir de la pieza: "'Rache' es una palabra alemana que significa 'Venganza', de modo que no pierda el tiempo buscando a una dama de ese nombre" (44).

También en Un enemigo de Napoleón, Holmes se sabe superior y no puede evitar reírse ante la simplicidad del razonamiento de Lestrade quien, contento con una burda explicación, no muestra señales de querer aclarar realmente el crimen, es decir, de llegar a la verdad. Allí Holmes le llama la atención con cierta vehemencia, recordándole que aun no ha explicado lo más importante, es decir, la destrucción de los bustos. 
Con eso queda suficientemente establecido que los policías sirven, ante todo, para destacar más aún los méritos y dotes intelectuales del detective privado. Los inspectores suelen tener buenas intenciones, ser hombres de acción que siguen sus intuiciones, pero carecen de teoría. ${ }^{23}$ Así son concebidos como figuras contrastantes que, con su falta de éxito, solo logran que se impongan el raciocinio y el pensar metódico, es decir las virtudes del héroe-detective.

Por otro lado, como señala Manuel Valle, la diferencia entre la policía y el detective queda enfatizada por la independencia que Holmes ostenta en la investigación de sus casos. Aunque es posible afirmar que comparte los fines de la policía, son sus métodos los que podrían calificarse de "no oficiales". En tal sentido, el investigador

no estaba vinculado con el crimen, sino con el misterio, [puesto que] el crimen es sólo una parte del misterio. De ahí que, para resolver el crimen (lo parcial), sea necesario remitirse siempre al misterio (el todo). Por ello, debido a la vinculación exclusiva que la policía tiene con el crimen/criminal, en numerosos relatos da por concluido el caso cuando tiene un 'culpable' para un 'crimen'. Pero el crimen no es —no tiene por qué serel 'núcleo' del misterio, y la solución del crimen, si deja cabos sueltos del misterio, terminará siempre por ser falsa (Valle 157).

Esta autonomía en el actuar del detective puede observarse también en que, pese a compartir los valores sociales de la legalidad y probidad con las instituciones oficiales, "[m]ore tan once he allowed the guilty perpretator to go free because overriding moral extenuating considerations and mitigating factors" 24 (Bagget[2]).

A pesar de esta gran diferencia entre el ojo privado y el ojo público, el detective privado colabora con los policías, a menudo empleándolos como sus instrumentos. Esta alianza adquiere su sentido más profundo por el hecho de que el detective se pone de esta forma abiertamente de

23 Según Sonja Osterwalder, “legt Holmes den grössten Wert auf die Logik als wichtigste Waffe seiner Methode und seines Denkens; gleichwohl wird er nie müde zu betones, dass Logik allein zu wenig erfolg führt. Ebenso wichtig sind Fantasie, Instinkt und Intuition" ("Holmes da gran importancia a la lógica como el arma más importante de su método y su pensamiento. Sin embargo, no se cansa en destacar que la lógica sola no basta para tener éxito. Igualmente importante son fantasía, instincto e intuición") (2011:71). Por eso, les reprocha a los policías que carecen casi sin excepción del "Gabe de Fantasie" ("don de la fantasía").

24 "[M]ás de una vez, permitió al culpable quedar libre gracias a extenuantes y esenciales consideraciones morales y a factores mitigantes". 
lado de la legalidad y del poder que protege las normas de la sociedad (cfr. Nusser 50). ${ }^{25}$ Tal oculta defensa del status quo revela que la crítica holmesiana a determinados grupos sectarios y a la policía es, en el fondo, más bien de una crítica social que de una crítica ideológica, dado que, según Rainov,

[e]l ambiente social y el clima histórico están ausentes casi, o totalmente, en la mayor parte de las producciones de Doyle. En dichas narraciones domina un vacío peculiar, carecen de todo lo que no esté relacionado directamente con el delito. Están impregnadas del helado aliento del crimen; en ellas no se distinguen personas vivas, sino que habitan personasesquemas, que cumplen puramente un papel oficial como elementos condicionados de un problema (67).

Aunque estas afirmaciones son algo exageradas, sobre todo con respecto a las figuras de Holmes y Watson, indican, sin embargo, que Doyle prácticamente no se interesa por una explicación sociológica o psicológica de los motivos (avaricia, venganza, codicia) de los criminales: "Die Kehrseite des von Holmes vertretenen 'Pathos der Faktizität' ist die Indifferenz allen moralischen Implikationes seinerm Tätigkeit gegenüber"26 (Nusser 99). Doyle no plantea problemas morales sino intelectuales, convirtiéndose así enteramente en un representante del relato de acertijo con su respectiva pérdida de realismo. Más que un crítico de la sociedad contemporánea, fue Holmes "un eminente filósofo de la ciencia", dedicado casi exclusivamente al "descubrimiento de la verdad" (Rehder 296-97). En este sentido, Cawelti señala que "[t]he detective's terrifying ability to expose hidden secrets also relates to the convention of aristocratic and eccentric detachment from the ordinary concerns of human life"27 (Cawelti 95). Esto se debe, según el mismo

Según Peter Nusser

[d]ie Ironie des überlegenen Detektivs, der einer ihm unterlegenen Polizei als Entschädigung für die Demütigung ihrer Beamten zuweilen am Ende den Lohn der Anerkennung, den sie nicht verdient, zuschiebt oder überlässt, ist nach Kracauer ein 'billiger Spass', weil sie über die 'gemeinsame Abhängigkeit' von den gesellschaftlichen Normen hinwegtäuscht und lediglich den Ruhm einer vom Gesellschaftlichen abgelösten ratio im Sinn hat. (50)

("[1]a ironía del detective superior, quien le ofrece a la policía derrotada al final del cuento el mérito del reconocimiento, que no merece, como recompensa para la humillación de sus empleados, es, según Kracauer, un 'chiste barato', porque engaña respecto al hecho de la 'dependencia común' de ambos de las normas sociales y persigue únicamente la fama de una razón separada de lo social").

26 "La otra cara del 'pathos de la facticidad' de Holmes es la indiferencia respecto a todas las implicaciones morales de sus acciones". 
autor, a la manera en los detectives y especialmente la figura de Sherlock Holmes evoca a la del famoso Sigmund Freud; en tanto ambos "are intelectual investigators of the illicit secrets of middle-class society. But where Freud and other social and psychological critics such as Marx and his followers discovered everyone's guils, Holmes and the other classical detectives absolved society by exposing the least-likely person or the master criminal"28 (Cawelti 96).

\section{Entre la ciencia y el sentimiento: Holmes y Watson, las dos caras del héroe}

Para destacar más aún la grandeza del filósofo de la ciencia y del gran detective Holmes, Doyle inventa, aparte de la ya mencionada policía, la figura del Dr. Watson, que es una fiel imagen tanto de su creador como del narrador anónimo de las historias de Dupin. Pero mientras el narrador de los cuentos de Poe era un simple nombre, "Watson es en cambio un personaje: sensato y sincero, alegre y valiente, bonachón y un tanto obtuso, participa de manera destacada en muchos lances y ha sido modelo de muchos personajes similares posteriores" (Del Monte 109-10). Mientras la amistad entre Dupin y el narrador nace, ante todo, porque ambos son de mentalidad melancólica y romántica y tienen una tendencia hacia lo macabro, la base de la amistad entre Sherlock Holmes y Dr. Watson es más racional: ambos son hijos de la época positivista y de las Ciencias Naturales. Por eso, no nos sorprende que ellos se conozcan en un laboratorio, mientras Dupin y su narrador-amigo lo habían hecho en una librería.

Watson se caracteriza, además, por ser un hombre de sociedad, un gentleman que se presenta desde el comienzo con una tarjeta de visita. Como doctor de Medicina es un profesional y como ex miembro del Cuerpo de Sanidad del ejército inglés pertenece al cuerpo de oficiales. A pesar de que no depende de un sueldo mensual, ejerce una profesión útil y mantiene una consulta que le deja suficiente tiempo libre (cfr. Suerbaum 52-54).

27 "la aterradora habilidad del detective de exponer secretos ocultos se relaciona también con la costumbre aristocrática y excéntrica de la indiferencia por los problemas ordinarios de la vida humana".

28 "son investigadores intelectuales de los secretos ilícitos de la clase media. Pero donde Freud y otros críticos sociales y psicológicos como Marx y sus seguidores descubren la culpabilidad de todos, Holmes y otros detectives clásicos absuelven a la sociedad al exponer a la prsona menos probable de ser culpable o a la mente maestra criminal". 
Como compañero del detective y narrador, Watson, al igual que el narrador de Poe, admira ${ }^{29}$ al gran detective y representa el pensamiento común, pero abierto a reconocer el carácter científico del método de Holmes (a diferencia de la policía). Como narrador y más aún como figura protagónica (por ejemplo, en El sabueso de los Baskerville), mantiene Watson una mayor cercanía con el público que el excéntrico Holmes. El lector crece en su autoestima cuando logra relacionar los cabos sueltos un poco más rápido que Watson, quien representa al good beefy Englishman. ${ }^{30}$ Llamarlo, por eso, el "ewiger Dummkopf"31 (Schklovskij 78), me parece una exageración. Más apropiada es probablemente la comparación con Sancho Panza. Finalmente, a diferencia del narrador de Poe, Watson "nimmt innerhalb der Handlung dem Übermenschen die Kleinarbeit ab und kann ihn häufig aus schwierigen Situationen (oft als deus ex machina) retten"32 (Buchloh y Becker 65).

Aún es posible agregar otra función importante en el rol del doctor Watson como narrador de los casos holmesianos. La empatía y la sensibilidad del personaje, más allá de permitir la conexión con el lector, construyen el relato de misterio de tal forma que el asombro y el reconocimiento estético puedan producirse de manera efectiva. Aunque Holmes no reconozca los méritos literarios de su compañero, argumentando que "el detectivismo es, o debería ser, una ciencia exacta, que es preciso tratar de la misma manera fría o antisentimental que toda ciencia exacta" (Conan Doyle, Estudio en escarlata), es la narrativa la que construye realmente la historia. Así, para Manuel Valle,

se establece, de esta forma, una verdadera paradoja, que constituye uno de los nudos gordianos más emocionantes de la narrativa policial: el detective/científico que se ocupa de desvelar los misterios, abomina del discurso narrativo en que aparecen esos misterios, pero -y aquí la paradoja sublime- es justamente ese discurso el que [...] produce tanto al detective

29 Sonja Osterwalder ve incluso en la admiración de Watson a Sherlock Holmes uno de sus "weibliche Züge" ("rasgos femeninos"). Otros serían, según ella, "seine sorgenvollen Bemühungen, ihn von der Drogensucht abzubringen" ("sus preocupaciones para que Holmes supere su drogadicción") y, ante todo, el hecho del "Ohnmacht, in die Watson fällt, als der totgeglaubte Detektiv zurückkehrt" ("desmayo, que afecta a Watson cuando ve al supuestamente muerto detective") (2011:59).

30 Kaymann afirma esta aseveración diciendo de Watson que representa "'the sturdy middle-clase virtues" ("las virtudes de la robusta clase media") (49).

31 "[E]terno estúpido".

32 "[A]sume los trabajos poco significativos dentro de la acción del superhombre y logra salvarlo frecuentemente de situaciones difíciles (a menudo como deus ex machina)". 
como al misterio. Digámoslo de una vez: sólo el relato 'watsoniano' -de Watson/Doyle- crea el misterio. Podemos, pues, concluir que el misterio es un efecto narrativo producido en -y por- el relato. (Valle 257)

No obstante, como el mismo Valle afirma, la preocupación central de Holmes está en el misterio, no en el crimen. Y si el misterio es la causa de un trabajo narrativo, el mismo detective se transforma en narrador. Para McInerny "[i]t is part of the artist in him that he sees human behavior as always story bound, and therefor interpretable in terms of the Aristotelian triad of beginning, middle and end. If he can get a good working sense of the first two, there is a fair chance that he can then figure out the third"33[3]. De tal forma, podemos afirmar que Holmes, aunque necesita a Watson y a su dimensión narrativa "sentimental" (Valle), al crear hipótesis y proponer soluciones a los casos es también un narrador de su propia historia ${ }^{34}$.

Por último, cabe mencionar la prevalencia de la figura de Sherlock Holmes en el gusto masivo. Si el detective resolvió sus primeros casos en periódicos, el día de hoy los resuelve principalmente en la televisión. Holmes se ha transformado en un héroe casi legendario y es la figura que inspira las historias detectivescas que se han hecho inmensamente populares en la pantalla chica o grande durante la última década ${ }^{35}$. Tal vez porque, como afirma Stephen Knight, "the imaginative and ideological forces realised in [Conan Doyle's] stories are real; in that respect Holmes is an archetype of the whole century's crime fiction"36 (62). Sin embargo,

33 "[E]s parte del artista en él que vea el comportamiento humano siempre ligado a una historia, y, por lo tanto, interpretable en los términos de la tríada aristotélica de principio, desarrollo y desenlace. Si puede entender el sentido de las dos primeras, hay una buena posibilidad de que pueda figurarse la tercera".

34 Esta cualidad de Holmes significa, según la interpretación de McInerny, que es posible descubrir una veta metafísica en el detective, por cuanto "he is familiar with thinking in terms of the cosmic whole and of thus recognizing that the kind of order and intelligibility that he readily recognizes on the level of everyday experience is founded on and ultimately explained by the order and intelligibility to be found in the universe itself" ("está familiarizado con el pensamiento en términos del absoluto cósmico y de ahí, reconocer que el tipo de orden e inteligibilidad que él reconoce en el nivel de la experiencia cotidiana, se encuentra y es explicado en última instancia en el universo mismo").

35 producciones directamente inspiradas en la figura de Conan Doyle son: Sherlock (2010), emitido por la cadena inglesa BBC, las mundialmente famosas películas de Guy Ritchie Sherlock Holmes (2009) y Game of Shadows (2011), con Robert Downey Jr. como protagonista, y la más reciente Elementary (2012), producida por la cadena norteamericana CBS. Sin embargo, cabe mencionar algunas otras series que claramente aluden al personaje del detective: House M.D (2004), de FOX, que hace especial hincapié en la relación Watson-Holmes; Bones (2005), de la misma cadena, que presenta a su protagonista, Temperance Brennan, como una especie de reescritura femenina de Holmes; por último C.S.I (2000) y Criminal Minds (2005) de CBS, que a través de la presentación de un equipo variado retratan las múltiples habilidades detectivescas de Holmes y la empatía y cercanía de Watson. 
la frialdad característica (matizada, eso sí, con algunos débiles destellos de empatía y de emocionalidad) y la actitud a menudo poco compasiva del detective hacen más plausible la afirmación de Marinaro y Thomas, "Holmes and Watson respectively represent the two essential elements that create a hero: greatness and goodness" ${ }^{\prime 37}$. No se trata de afirmar que el protagonista de Doyle carece en absoluto de bondad o favorece las fuerzas del mal: por el contrario, su probidad y colaboración con las nociones de ley y justicia son innegables. Sin embargo, la vocación de servicio y la entrega a otros que se requiere para tener un carácter realmente heroico (según la lectura de Carlyle, analizada por las autoras), no está realmente exacerbada en la figura del detective. De tal forma “Sherlock Holmes possesses Carlyle's 'eyesight' but lacks the emotional perception of Dr. Watson"38 (Marinaro y Thomas[4] ).

En efecto, Watson, militar retirado, que ofrece su salud al servicio del Imperio y que suele tener conductas caballerosas, galantes y consideradas, a menudo quedaba escandalizado con el pragmatismo de su compañero, que ve los crímenes como un estimulante mental y casi siempre pierde interés en sus clientes una vez terminado el caso. Aun así, el atractivo del personaje de Holmes es innegable, como las múltiples producciones inspiradas en los casos de Baker Street confirman. Tal vez, la falta de sensibilidad del detective se transforma en la plataforma para crear situaciones cómicas (famoso es el humor negro de "Dr. House", personaje reconocidamente inspirado en Holmes). Sin embargo, es evidente que en todas las reelaboraciones y reescrituras mediáticas de la obra de Doyle uno o más personajes se reparten el rol del buen doctor y matizan la fría ciencia con alguna historia sentida, cercana al corazón, o mostrando empatía hacia las víctimas. De tal forma, es posible afirmar con Marinaro y Thomas que el héroe arquetípico que ha trascendido en la cultura de masas es un híbrido con dos caras: una científica y desapegada (Holmes), y una sentimental y humana (Watson).

A través de todas estas funciones de su narrador Watson, comprueba Doyle nuevamente, hasta qué punto ha asimilado el modelo de Poe que

36 "Las fuerzas imaginativas e ideológicas materializadas en las narraciones de Conan Doyle son reales; en este sentido, Holmes es un arquetipo de la ficción criminalística de todo el siglo".

37 "Holmes y Watson representan, respectivamente, los dos elementos esenciales para crear un héroe: grandeza y bondad".

38 "Sherlock Holmes posee la visión de Carlyle pero no tiene la percepción emocional del Dr. Watson". 
hibridiza con bastante creatividad, mezclando sus propios intereses temáticos con los de su precursor y estableciendo, así, el modelo del género policial más imitado en la historia.

\section{Bibliografía}

Bagget, David. "Sherlock Holmes as Epistemologist". The Philosophy of Sherlock Holmes. Por Bagget, David y Phillip Tallon, eds. Kentucky: The University Press of Kentucky, 2012. Medio electrónico.

Boileau-Narcejac. La novela policial. Buenos Aires: Paidos, 1968.

Bonfantini, Massimo A. y Giampaolo Proni. “To Guess or not to Guess?” El signo de los tres. Dupin, Holmes, Peirce. Por Umberto Eco y Thomas A. Sebeok, eds. Traducción: E. Busquets. Barcelona: Lumen, 1989. 164-184.

Buchloh, Paul G. y Jens P. Becker. Der Detektiv-Roman. Studien zur Geschichte und Form der englischen und amerikanischen Detektivliteratur. Cuarta edición. Darmstadt: Wissenschaftliche Buchgesellschaft, 1990.

Cawelti, John G. Adventure, Mystery, and Romance. Formula stories as Art and Popular Culture. University Chicago Press, 1976.

Del Monte, Alberto. Breve historia de la novela policiaca. Madrid: Taurus, 1962.

Doyle, Arthur Conan. Estudio en escarlata. Traducción: Alvaro Delgado Gal. Sexta reimpresión. Madrid: Alianza, 1997.

---. El sabueso de Baskersville. Santiago de Chile: Andrés Bello, 2005.

---. "La liga de los pelirrojos". La liga de los pelirrojos. Por Arthur Conan Doyle. Santiago de Chile: Zig-Zag, 1945. 7-42.

---. "El enemigo de Napoleón". Aventuras de Sherlock Holmes. Por Arthur Conan Doyle. Santiago de Chile: Zig-Zag, 2012. 22846. 
[5]Eco, Umberto. "Cuernos, cascos, zapatos: Algunas hipótesis sobre tres tipos de abducción." El signo de los tres. Dupin, Holmes, Peirce. El signo de los tres. Dupin, Holmes, Peirce. Por Umberto Eco y Thomas A. Sebeok, eds. Traducción: E. Busquets. Barcelona: Lumen, 1989. 265-294.

Kaymann, Martin A. "The short story from Poe to Chesterton". The Cambridge Companion to Crime Fiction. Por Martin Priestman, ed. Cambridge (UK): Cambridge University Press, 2003. 41-58.

Knight, Stephen. "Detective Apoteosis: Sherlock Holmes". Crime fiction 1800-2000. Nueva York: Palgrave Macmillan, 2004. 55-63.

Marinaro, Francesca y Kayley Thomas. "'Don't Make People into Heroes, John': (Re/De) Constructing the Detective as Hero". Sherlock Holmes for the 21st Century. Essays on New Adaptations. Por Porter, Lynette, ed. North Carolina: McFarland \& Company, Inc., 2012. Medio electrónico.

McInerny, D. Q. “Sherlock Holmes: Artist of reason”. The Philosophy of Sherlock Holmes. Por Bagget, David y Phillip Tallon, eds. Kentucky: The University Press of Kentucky, 2012. Medio electrónico.

Nusser, Peter. Der Kriminalroman. Stuttgart: Metzler, 1980.

Osterwalder, Sonja. Düstere Aufklärung. Die Detektivliteratur von Conan doyle bis Cornwell. Wien . Köln .Weimar: Böhlau Verlag, 2011.

Rainov, Bogomil. La novela negra. La Habana: Editorial Arte y Literatura, 1978.

Rehder, Wulf. "Sherlock Holmes, detective filósofo". El signo de los tres. Dupin, Holmes, Peirce. Eds. Umberto Eco y Thomas A. Sebeok. Trad.: E. Busquets. Barcelona: Lumen, 1989. 295-312.

Schklovskij, Viktor "Die Kriminalerzählung bei Conan Doyle." Der Kriminalroman I. 76-94. 
Schulz-Buschhaus, Ulrich. Formen und Ideologien des Kriminalromans. Ein gattungsgeschichtlicher Essay. Francfort del Meno: Athenaion, 1975.

Scaggs, John. Crime Fiction. London y Nueva York: Routledge, 2005.

Sebeok, Thomas A. y Jean Umiker-Sebeok. "'Ya conoce usted mi método': Una confrontación entre Charles S. Peirce y Sherlock Holmes." El signo de los tres. Dupin, Holmes, Peirce. Eds. Umberto Eco y Thomas A. Sebeok. Trad.: E. Busquets. Barcelona: Lumen, 1989. 31-81.

Symons, Julian. From the Detective Story to the Crime Novel. Mysterious Press Books, New York, 1993.

Skreb, Zdenko. "Die neue Gattung. Zur Geschichte und Poetik des Detektivromans." Der wohltemperierte Mord. Por Victor Zmegac, ed. Francfort del Meno: Atnenäum, 1971. 35-96.

Smuda, Manfred. "Variation und Innovation." Der Kriminalroman I. Por Jochen Vogt, ed. Munich: Fink, 1971. 33-62.

Suerbaum, Ulrich. Krimi. Eine Analyse der Gattung. Suttgart: Reclam, 1984.

Truzzi, Marcello. "Sherlock Holmes: Experto en psicología social aplicada." El signo de los tres. Dupin, Holmes Peirce. Por Umberto Eco y Thomas A. Sebeok, eds. Traducción: E. Busquets. Barcelona: Lumen, 1989.

Valle, Manuel. El signo de los cuatro. Tomo I: Conan Doyle, la ciencia como ficción sentimental. Granada: Comares, 2006. 